\title{
A Wireless Sensor Network Based on Unmanned Boundary Sensing Technique for Minimizing Human Elephant Conflicts
}

\author{
Jerline Sheebha Anni DHANARAJ ${ }^{1 *}$, Arun Kumar SANGIAH ${ }^{2}$ \\ ${ }^{1}$ Research Scholar, School of Computer Science and Engineering (SCOPE), \\ VIT University, Vellore, 632014, India \\ jerline_dhanaraj@yahoo.co.in (*Correspondingauthor) \\ ${ }^{2}$ Associate Professor, School of Computer Science and Engineering (SCOPE), \\ VIT University, Vellore, 632014, India \\ sarunkumar@vit.ac.in
}

\begin{abstract}
Unmanned boundary sensing technique targets to preserve and protect both the elephant breed and the human inside their protected areas without crossing the boundary. This paper proposes a novel integrated wireless sensor network architecture for elephant tracking. For this purpose, the boundary sense utilizes the most advanced sensors including vibration sensors, acoustic sensors and camera sensors, as these three sensors mutually balance their demerits leading to a promising solution. The architecture to deploy and activate boundary sensing is presented in this paper. The system offers three variations of detections using vibration, sound and image, and assesses them on a massive dataset of real time recordings. Moreover, the system integrates these sensors and fulfills the requirements, which is not adequately addressed in the existing literature. Based on this context, the detector is further enhanced based on the distance metric, SHORT, MEDIUM and LONG, and determined through a fuzzy inference system. Finally, the overall performance evaluation of the proposed system achieves $97 \%$ detection rate and $0 \%$ false alert for elephant detection.
\end{abstract}

Keywords: Boundary sensing technology, Elephant tracking, Fuzzy interference system, Distance metric.

\section{Introduction}

The remedy of Human-Elephant Conflict (HEC) is a swiftly escalating region of research across the globe, where conservationists are functioning hard, to identify the solution, under which pressures are towering high between people (located near forest boundary especially villagers) and their elephant neighbors. Scientists and engineers are working relentlessly to discover a way out from such conflict. Earlier studies [4] have reported elephant tracking using wireless sensor networks (WSN) as the best option for minimizing the HEC. WSNs are of low cost and allow coverage of a specified area with a network of simple devices. Importantly, they offer an obtrusive solution where elephants are not forced to carry any electronic devices. Many sensors such as acoustic, seismic, and passive infrared (PIR) sensors consume small power. Out of these, seismic sensor, acoustic sensor and camera sensor have played a vital role in elephant detection. Because sound, vibration and image identification are the only three ways for tracking the elephant. A brief introduction of the usage of these sensors for elephant detection is provided.

Seismic sensors are also called as vibration sensors are used to measure seismic vibrations of elephants by converting ground motion into a measurable electronic signal, used for various applications in elephant tracking such as classification of species, census counting and also act as an early warning system. The challenges in this detection setup, constitute a chance of insufficient vibrations observed by the geophones, due to natural calamities like rain and soil humidity. Hence a failure may occur in the detection of elephant movement. Therefore, a protected hardware setup that is robust against environmental changes and natural hazards is required for efficient spotting of the occurrence of elephant vibrations.

In many applications, acoustic sensors provide vigorous automated detection of elephant vocalizations. The research on acoustic analysis of elephant rumbles has particularly addressed tasks, such as are early warning system for elephants, identification of elephants by their calls and the analysis of particular call types. The challenge for the automated detection of elephant vocalizations is robust to the multiple noise sources. On the other hand, elephant vocalization recordings frequently enclose unnecessary sounds, including other wild animal sounds, breeze, elephants walking through the hedgerow of bushes or shrubs and other noises that occur in the forest.

Camera sensors work towards image detection of elephants. Various scientists and conservationist have used cameras equipped with infrared 
triggers, called camera traps, to obtain critical data about wildlife and their habitats. Cameratrapping is a useful and widely used tool to study wildlife. Camera-traps are ideally suited to sample medium to large sized, ground-dwelling mammals. Hence best suitable for elephants. The important issue which has been addressed is the inclusion of camera sensor is poor lighting and background. Integration of WSN architecture has been developed for tackling this problem.

Against this background, this paper proposes an efficient and long lasting WSN based system for elephant detection, by integrating these sensors. Existing studies used WSN system for HEC, but resulted in temporary solution because of various problems. The novelty of this paper is enhanced by using a hybrid WSN system. According to the result analysis, Hybrid sensor network provides a best solution for minimizing Human Elephant Conflict. To the best of our knowledge, this paper presents perhaps the first attempt that combines seismic, acoustic and camera sensors together, offering the best solution to HEC [1]. A combined vibration, acoustic and camera sensors provides a promising solution due to mutual compensation of their weaknesses between those techniques, leading to a highly reliable detection [2].

Elephant detection is efficiently detected using these sensors. However, the target position measurements are rarely uncertain due to unwanted noise, background images, lightings and weather conditions. The use of expert knowledge is used for minimizing the uncertainty. The advantage of fuzzy logic controller is its aptitude to deal with nonlinearities and uncertainties. Fuzzy inference system using fuzzy If-then rules are the efficient sources for expressing knowledge from experts. In this paper, a fuzzy filter with two linguistic variables namely prediction_success and prediction_failure are designed in order to maximize the detection rate and reduce the false alert.

Elephant passages are identified and this setup is installed to observe the progress of these herds. On response of the finding, the controller sends an alert to the forest authorities indicating detection of an elephant in the forest boundary. It is then the responsibility of the forest officials to chase the elephant back to the forest. The elephant detection is achieved without human intervention, hence our proposed system will act as unmanned boundary sensing approach.
This proposed system is explained in larger depth in the rest of this paper which is organized as follows. Section 2, provides overall system architecture. Section 3 presents the description of the methods Section 4 explains the experimental setup. Section 5 consolidates the different detectors along with their result analysis. The background and structure of fuzzy logic inference system are described in depth in Section 6. Finally, Section 7 lays down the conclusions.

\section{System Architecture}

The architecture of the elephant monitoring system is sketched in Figure 1. The architecture serves as a frame for the identification of the necessary components. The sketched system consists of five modules: (a) input module with different sensors, (b) processing module where the sensor records is analyzed using different algorithms, (c) detector module that provides analysis results with distance metric ranges for optimization, (d) fuzzy interference module where the outcomes of different sensors are integrated and fuzzified, and (e) output module that provides the integrated fuzzy based on analysis results.

The sensor inputs can be exceptionally multimodal consisting of acoustic sensors, camera sensors and vibration sensors. The processing module consists of three detectors (acoustic detector, vibration detector and visual detector), where detection is performed. The detections form the basis and furthermore serve as inputs to higher level tasks in the overlying fuzzy interference module. Basic Advantage of Fuzzy Logic Controller is it allows different sources to merge uncertain information in a best way and incorporate heuristic control in the form of if-then rules. Hence suitable for our proposed unmanned boundary sensing system.

\section{Research Methodology}

\subsection{Data Collection}

\subsubsection{Study Area}

Studies were made in the Hosur forest division, Tamil Nadu, India. The data were collected from various sources like the Hosur forest division websites, conversations with village people located near the forest boundary, elephant attack surveys and related literature review. Hosur region is abundantly artistic with hills, thick forests and bigger wildlife. 


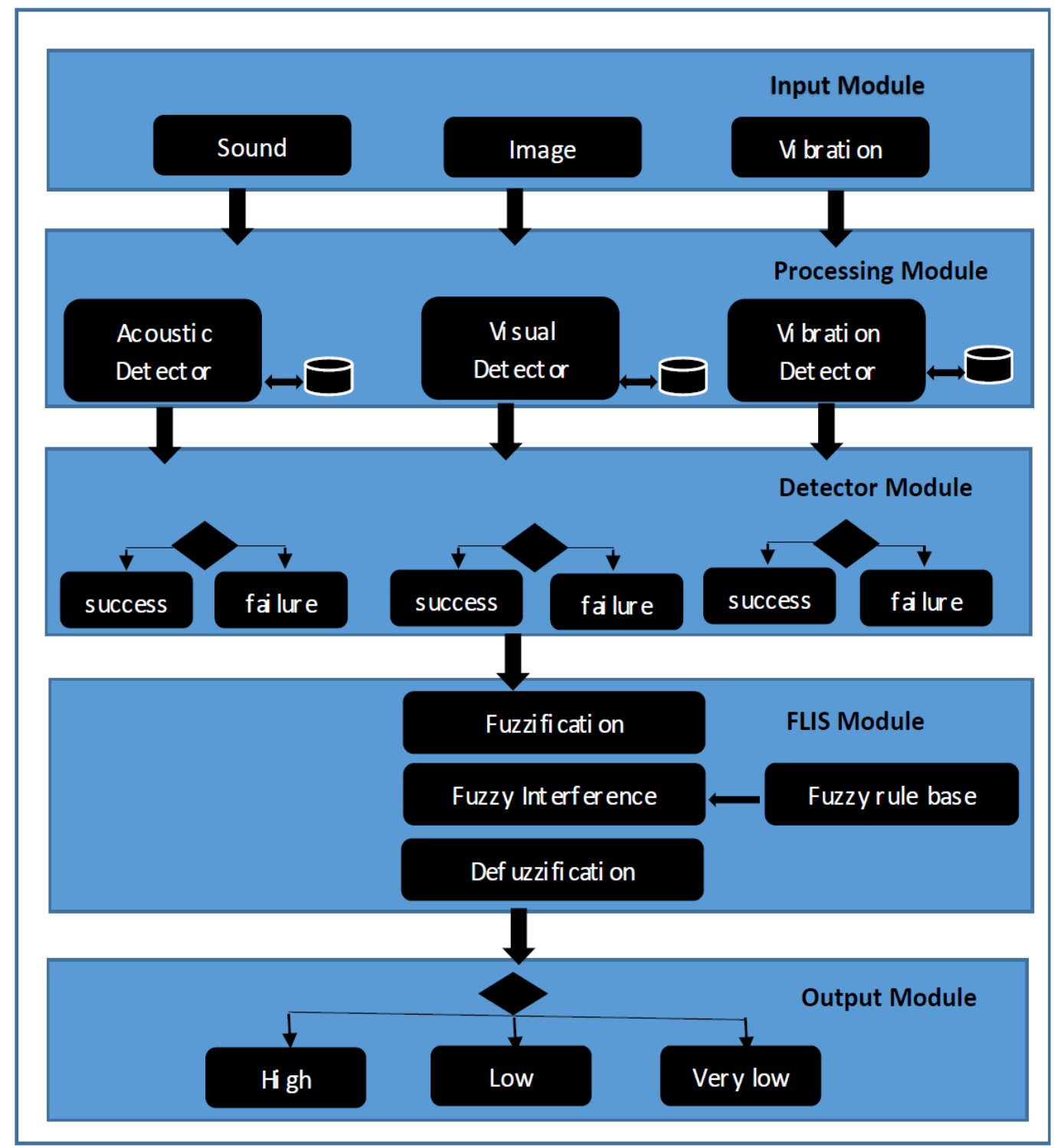

Figure 1. Schematic diagram of the proposed integrated WSN for Elephant Detection

\subsubsection{Recordings Context}

Fifty five hours of recordings were made between November 2014 and October 2015 in the southern part of Hosur forest area, Tamil Nadu. Experimental sessions were carried out in spatial separation and reunion social contexts, which rarely involved commands by the keepers. In this context, the following are the four targeted focal elephants (based on age/ gender classification) female young, female adult, male young and male adult.

The focal elephant was isolated while the remaining elephants were taken out of sight by the keeper (600-750m) away. The focal elephant was then recorded in different distances inside the sensor unit. For the reunion context, all four elephants were reunited one by one and recorded in different distances inside the sensor unit.

\section{Experimental setup}

The training set in our experiments contains real time recordings of rumbles, vibrations and images of Elephants. The data set includes 505 elephant vocalizations, 371 vibrations and 625 elephant images. Acoustic recordings were prepared using a Neumann TLM-10B microphone and a TASCAM HD-P2 recorder. The elephant acoustic detector includes, a database consisting of 505 vocalizations and 133 background sounds and other noises used as a negative training set. The hardware setup including wireless camera was prearranged to capture the image of elephants. The wireless camera was fixed on the stem of a 
tree. Using a RF receiver, the video received was converted into image frames using a camcorder software in PC. The elephant visual detector includes a database consisting of 625 images and 156 randomly chosen images of other animals are used as a negative training set. Vibration recordings were made of the pachyderm footfalls as they passed close to the geophone LGT 2108. A pre-amplifier was used to rise the recording gain, a sound card used for converting the signal from analogue to digital, which was then verified on a Dell Inspiron laptop using the cool edit pro version 1.2. An elephant vibration detector sets the threshold based on the sensor output.

\subsection{Single Node}

The experimental setup consists of a Camera, a Vibration Sensor and an Acoustic Sensor. These three sensors are deployed in the node $\mathrm{S}$ as shown in Figure 2.

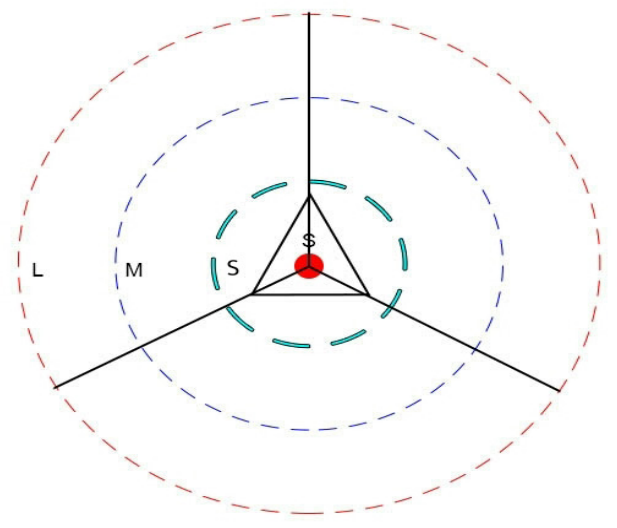

Figure 2. Single sensor unit

The ranges of the regions are mentioned as Short (S), Medium (M), Long (L). The distance of S, M, $\mathrm{L}$ are denoted as $\mathrm{X}, \mathrm{Y}$ and $\mathrm{Z}$. Here the distance of $\mathrm{S}, \mathrm{M}, \mathrm{L}$ are same i.e., if distance of $\mathrm{X}=5 \mathrm{~m}$, then $\mathrm{Y}=5 \mathrm{~m}$ and $\mathrm{Z}=5 \mathrm{~m}$.

$$
X=Y=Z
$$

Initially the setup $\mathrm{S}$ has a default value of 0 . If the elephant enters into the sensor region the following actions occurs:

1. When the elephant enters the region of $\mathrm{Z}$, then the value of camera, Vibration Sensor and Acoustic Sensor is Long (L).

2. When the elephant enters in region $\mathrm{Y}$, the value of all three is denoted as Medium (M).

3. When the elephant enters in the region $\mathrm{X}$, the value of all three is changed as Short (S).
The above mentioned are normal conditions i.e., when camera, vibration sensor and Acoustic sensors work correctly. If any one or two of the setup does not work sound it shows its lastly stored value. Thus conflict occurs in these conditions.

\subsection{Multi Node}

The nodes are created in the parallel format for overcoming this conflict. The distance of placing another node is calculated as follows:

$$
X+Y+Z=A
$$

i.e. if the distance of $X=5 \mathrm{~m}$ then the next node is placed in the distance of $10 \mathrm{~m}$. Let us consider two nodes $\mathrm{S} 1$ and $\mathrm{S} 2$. The range of $\mathrm{S} 1$ and $\mathrm{S} 2$ are interfered as follows:

The failure of one or two of the setup can be easily detected by analyzing the neighbor nodes as shown in Figure 3. Due to the small dimension of a test sensor unit, the power supply attached to the sensor nodes has to be very limited in size. Always energy conservation becomes a challenging issue in any WSN unit. However, batteries, as the primary power supply, still fail earlier in some sensor unit than their projected working time. Meanwhile, it is a challenge for researchers and developers to obtain long operating hours without sacrificing the system performance.

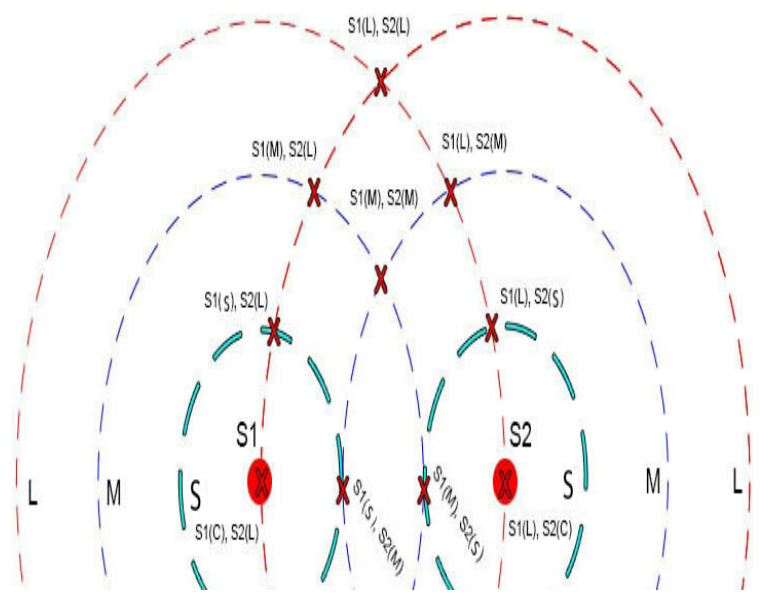

Figure 3. Dual Sensor Unit

\section{Experiments and Results}

This section includes description about acoustic detector, vibration detector and visual detector along with its result analysis. 


\subsection{Acoustic Detector}

Utmost acoustic analysis researches on elephant vocalizations addresses specific tasks like vocal identification of individual elephants [8], analysis of particular call types [9] and intra-call variations [10], [11]. The automated detection of elephant vocalizations has rarely been inspected so far. Acoustic detection of elephants was performed by Matthias [7]. The author detected the elephants from their rumbles which improves the robustness of the detector even in noisy situations and achieves $88.2 \%$ detection rate and $13.7 \%$ false alert.

The Neumann Microphone is ideally suited for the proposed acoustic elephant detector, for capturing the environmental signal from all directions explained in Section 3. The captured signals act as inputs for the proposed acoustic elephant detector. Recording quality purely depends on the distances between the elephant and the microphone. Spectrograms are used for finding the rumbles by visual inspection with respect to time. The main objective of the proposed work is to discriminate the elephant sound from other noise sources like noise initiated by other animals, wind, rain and other low-frequency disturbers like engine sounds of vehicles using filters. The filtered input vocalization signal is further compared with the recorded vocalization stored in the database. When a match is found, the detector sets the target as detected.

\subsubsection{Acoustic Detector: Result Analysis}

The proposed acoustic elephant detector achieves $88.0 \%$ detection rate and $13.5 \%$ false alert. Our conclusion tends to agree with those of Matthias et. al [7] differing only in materials and data set used by Matthias [7]. We found evidence for almost a similar percentage on the detection rate and false alert. The fuzzy ranges of acoustic detector are listed in Table 1.

Table 1. Fuzzy ranges of acoustic detector

\begin{tabular}{|l|l|l|l|}
\hline $\begin{array}{l}\text { Elephant } \\
\text { vocalization } \\
\text { from data } \\
\text { set }\end{array}$ & $\begin{array}{l}\text { Distance } \\
\text { from the } \\
\text { sensor } \\
\text { unit }\end{array}$ & $\begin{array}{l}\text { Fuzzy } \\
\text { range }\end{array}$ & $\begin{array}{l}\text { Linguistic } \\
\text { Values }\end{array}$ \\
\hline 203 & $15 \mathrm{~m}$ & 3 & LONG \\
117 & $10 \mathrm{~m}$ & 2 & MEDIUM \\
125 & $5 \mathrm{~m}$ & 1 & SHORT \\
60 & $0 \mathrm{~m}$ & 1 & SHORT \\
\hline
\end{tabular}

\subsection{Vibration Detector}

Existing research on vibration analysis of elephant addresses highly discerning responsibilities like census counting of elephants [5], species classification [6] and to monitor elephants in remote areas [2]. The automated detection of elephant vibrations has rarely been investigated so far. An early warning system for elephant intrusion in forest border areas was performed by S. J. Sugumar et al. [13]. The author detected the elephants from their vibrations and sends alerts to forest authorities by achieving $91.25 \%$ accuracy rate with $0 \%$ false alert. Seismic recordings can be made effective using vertical geophones. Hence, the geophone sensor system is ideally suited for the proposed elephant detector, due to its potential differentiation between an elephant footfall and other wild species footfalls.

The elephant footfall is adequately longer and contains more energy. O'Connell et al. [14] report the similarity between the frequencies of the wavelets of different species, but the duration of the elephant wavelet is longer. Each individual sensor has a ground signal coverage range with a radius of about 24m, say Sugumar et al. [13] and Campan [3]. Elephant's walking in the sensing range of the geophones produce vibrations. The captured vibrations will be acting as inputs for the proposed elephant vibration detector. The geophone when sensing a vibration generates a signal in millivolts $(\mathrm{mV})$. The output of geophone is amplified and compared with a threshold value. The threshold value is obtained according to the output caused by the vibrations produced by an elephant and group of elephants in a specified range.

\subsubsection{Vibration Detector: Result Analysis}

The proposed vibration elephant detector achieves a $91.2 \%$ detection rate with a $0 \%$ false alert. Our conclusion tends to agree with the conclusions of S. J. Sugumar et al. [12], differing only in materials and data set used by S. J. Sugumar et al. [13]. We found evidence for an almost similar percentage on detection rate and false alert. The fuzzy ranges of vibration detector are listed in Table 2.

\subsection{Visual Detector}

Visual detection is complementary to acoustic and vibration detection and may improve reliability. Existing research on visual analysis of elephant 
addresses a fully automated detection of elephant images and videos performed by Zeppelzauer et al. [15]. The author detected the elephants from their image by achieving a $91.7 \%$ accuracy rate with a $2.5 \%$ false alert (near) and $88.0 \%$ accuracy rate with a $39.0 \%$ false alert (far).

The aim is to get high quality and a close image of elephants. This proposed visual detector helps to detect the pachyderm and its group even in the presence of other wild species without any confusion. The cameras (rose on trees) capture the species image in the sensor unit. The captured image will be acting as the input image for the proposed elephant visual detector.

\subsubsection{Visual Detector: Result Analysis}

The proposed visual elephant detector achieves $91.7 \%$ detection rate with $2.5 \%$ false alert (near) and $87.9 \%$ accuracy rate with $39.0 \%$ false alert (far). Our conclusion tends to agree with the conclusions of Zeppelzauer et al. [15], differing only in materials and data set used by Zeppelzauer et al. [15]. We found evidence for almost a similar percentage on the detection rate and the false alert. The fuzzy range of visual detector is listed in Table 3.

\section{Fuzzy Logic Inference System}

\subsection{Background and FLIS Structure}

The varying result of different sensors needs to be finalized with mechanization and a step towards it, Mamdani fuzzy inference system was chosen as a technique for decision making and to finalize the results of acoustic, vibration and visual detectors.
In this paper, we recommend FLIS, a structural design for the effectual detection of elephant using integrated WSN based on fuzzy logic. For the first time, an automated elephant detection framework with fuzzy system that can finalize decisions based on the outputs obtained from the integrated WSN was developed.

\subsection{Fuzzy Linguistic Variables (LV) and Membership Functions (MF)}

Each linguistic value of a linguistic variable is related with a membership function which takes values between 0 and 3 as described in Table 4 .

In FLIS, there are two input linguistic variables Prediction_Success and Prediction_Failure and one output linguistic variable Probability of_Occurrence. The aim of FLIS is to infer numerical values for the output linguistic variable Probability_of_Occurrence from the numerical values of the input linguistic variables Prediction Success and Prediction_Failure.

The linguistic variables Prediction_Success can take linguistic values of SHORT, MEDIUM or LONG, Prediction_Failure can take linguistic value DISCARD and Probability_of_Occurrence can take linguistic values of HIGH, LOW or VERY LOW. Prediction_Success is estimated by the distance metric of elephant presence in the sensor unit. Prediction_Failure is estimated as non-detection of elephant by any sensors in the sensor unit. A sample of membership functions for the linguistic values of Prediction Success, Prediction_Failure and Probability_of_Occurrence is shown in Figure 4.

Table 2. Fuzzy Ranges of Vibration Detector

\begin{tabular}{|l|l|l|l|l|}
\hline Context & $\begin{array}{l}\text { Distance from } \\
\text { the sensor unit } \\
\text { in meters }\end{array}$ & $\begin{array}{l}\text { Vibration } \\
\text { sensor output in } \\
\mathbf{m V}\end{array}$ & $\begin{array}{l}\text { Fuzzy } \\
\text { range }\end{array}$ & $\begin{array}{l}\text { Linguistic } \\
\text { Values }\end{array}$ \\
\hline $\begin{array}{l}\text { Spatial } \\
\text { Context }\end{array}$ & $15 \mathrm{~m}$ & $6.3 \pm 0.5$ & 3 & LONG \\
& $10 \mathrm{~m}$ & $7.7 \pm 0.2$ & 2 & MEDIUM \\
& $5 \mathrm{~m}$ & $8.0 \pm 0.6$ & 1 & SHORT \\
& $0 \mathrm{~m}$ & $9.2 \pm 0.5$ & 1 & SHORT \\
\hline Reunion & $15 \mathrm{~m}$ & $10.4 \pm 0.4$ & 3 & LONG \\
Context & $10 \mathrm{~m}$ & $13.5 \pm 0.3$ & 2 & MEDIUM \\
& $5 \mathrm{~m}$ & $14.3 \pm 0.6$ & 1 & SHORT \\
& $0 \mathrm{~m}$ & $16.2 \pm 0.4$ & 1 & SHORT \\
\hline
\end{tabular}

http://www.sic.ici.ro 
Table 3. Fuzzy ranges of visual detector

\begin{tabular}{|c|c|c|c|}
\hline $\begin{array}{c}\text { Elephant images } \\
\text { from data set }\end{array}$ & $\begin{array}{c}\text { Distance from the } \\
\text { sensor unit }\end{array}$ & Fuzzy range & Linguistic Values \\
\hline 193 & $15 \mathrm{~m}$ & 3 & LONG \\
207 & $10 \mathrm{~m}$ & 2 & MEDIUM \\
183 & $5 \mathrm{~m}$ & 1 & SHORT \\
42 & $0 \mathrm{~m}$ & 1 & SHORT \\
\hline
\end{tabular}

Table 4. Scaling pattern

\begin{tabular}{|l|l|l|}
\hline \multicolumn{3}{|c|}{ Scaling Pattern } \\
\hline LinguisticVariables & Fuzzy Ranges & Linguistic Values \\
\hline \multirow{3}{*}{ Prediction_Success } & 3 & SHORT \\
\cline { 2 - 3 } & 2 & MEDIUM \\
\cline { 2 - 3 } & 1 & LONG \\
\hline Prediction_Failure & 0 & DISCARD \\
\hline
\end{tabular}

The shape of a membership function rivals human knowledge based on a specific application. Membership functions can be constructed by answering the following questions:

- To what range elephant presence is considered as SHORT?

- To what range elephant presence is considered as MEDIUM?

- To what range elephant presence is considered as LONG?
Answers to those questions help definition of pairs of numerical values and the distance that these values represent (SHORT, MEDIUM or LONG) as also forming the linguistic sets and corresponding membership functions. The membership functions can be constructed using curve-fitting methods. Use of trapezoidal and triangular shapes for membership functions is a common practice in fuzzy logic applications, due to their computational efficiency, such as those presented in Figure 4.

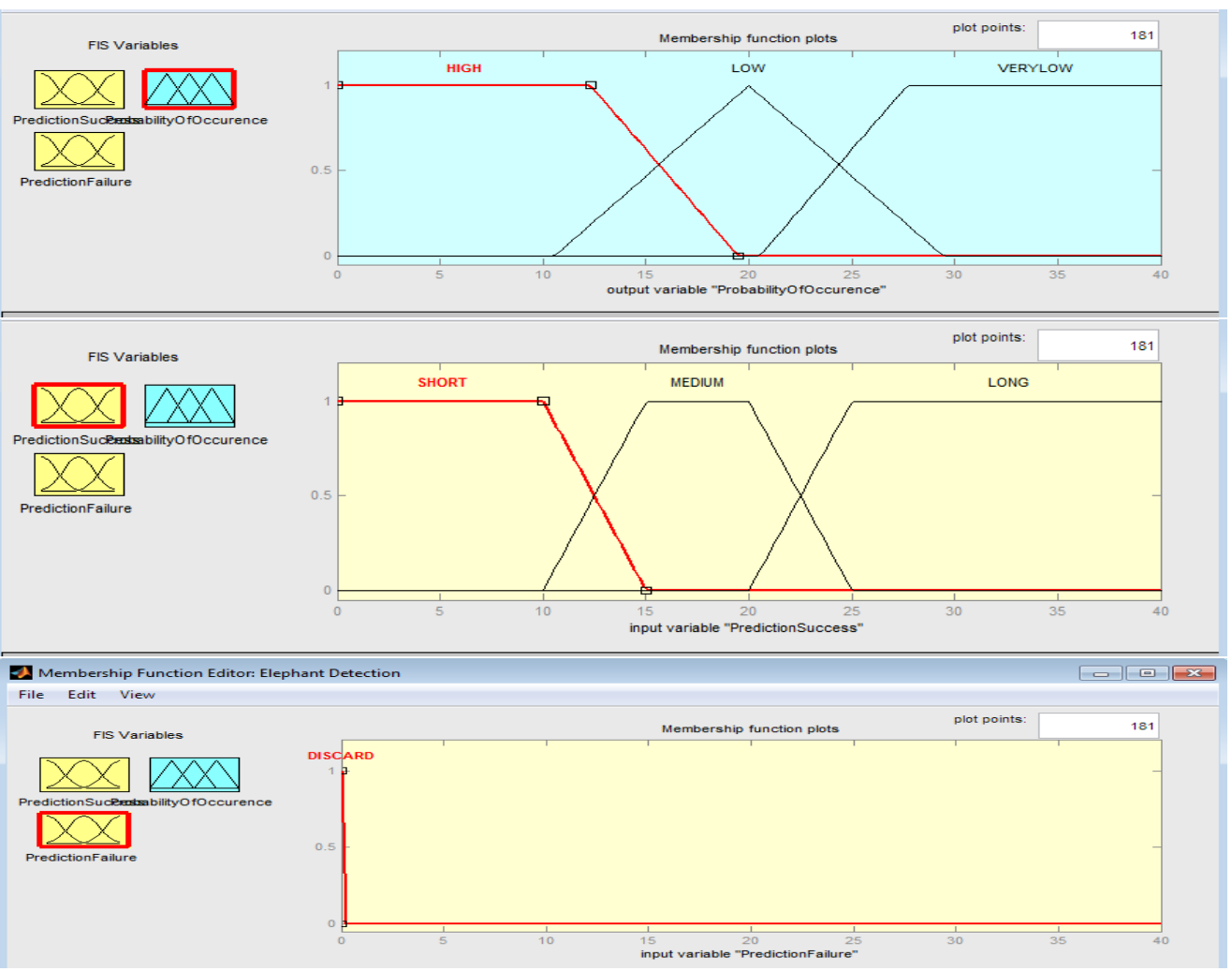

Figure 4. Membership Functions 


\subsection{Fuzzification and Fuzzy Rule Base}

The detector module gathers info from all three sensors and computes numerical values of Prediction_Success and Prediction_Failure. The fuzzification process converts these values to linguistic sets. These rules are constructed by integrating the expertise on classification of elephant location threshold as SHORT, MEDIUM or LONG.

Table 5 considers one sensor (S1) results in Prediction_Success and the other two sensors (S1 \& S3) resulting in Prediction_Failure.

1. If $\mathrm{S} 1$ results as short, then regardless of the results $\mathrm{S} 2 \& \mathrm{~S} 3$, the $\mathrm{P}(\mathrm{O})$ is set to high.

2. If $\mathrm{S} 1$ results as medium, and $\mathrm{S} 2$ and $\mathrm{S} 3$ resulted as discard, then the $\mathrm{P}(\mathrm{O})$ is set to low.

3. If $\mathrm{S} 1$ results as long, and $\mathrm{S} 2 \& \mathrm{~S} 3$ resulted as discard, then the $\mathrm{P}(\mathrm{O})$ is set to very low.

Table 6 considers two sensors (S1 \& S2) results in Prediction_Success and one sensor (S3) results in Prediction_Failure.

1. If $\mathrm{S} 1$ is short and $\mathrm{S} 2$ is short and $\mathrm{S} 3$ is discard, then $\mathrm{P}(\mathrm{O})$ is high.

2. If $\mathrm{S} 1$ is medium and $\mathrm{S} 2$ is medium and $\mathrm{S} 3$ is discard, then $\mathrm{P}(\mathrm{O})$ is low.

3. If $\mathrm{S} 1$ is long and $\mathrm{S} 2$ is medium and $\mathrm{S} 3$ is discard, then $\mathrm{P}(\mathrm{O})$ is very low.

Examples of other rules in Table 6 can be interpreted similarly.

Table 7 considers all three sensors (S1, S2 \& S3) resulting in Prediction_Success. As an example of these rules illustrated in Table 7.

1. If anyone sensor $(\mathrm{S} 1 / \mathrm{S} 2 / \mathrm{S} 3)$ results as short, then regardless of the results of others, the probability of occurrence is set to high.

2. If any combination of sensors results in Medium and/or long values, then $\mathrm{P}(\mathrm{O})$ is set to low or very low.

If one or two sensors are not working the system will not halt, which is described in Table 5 and Table 6. If all three sensors are working, the possible results are shown in Table 7 . If all the three sensors are not working then the system will halt, even then it achieves zero false alert.
Table 5. Fuzzy rules in case of two sensors failure

\begin{tabular}{|c|c|c|c|}
\hline \multirow{2}{*}{$\begin{array}{c}\begin{array}{c}\text { Prediction } \\
\text { Success }\end{array} \\
\text { S1 }\end{array}$} & \multicolumn{2}{|c|}{$\begin{array}{c}\text { Prediction } \\
\text { Failure }\end{array}$} & \multirow{2}{*}{$\begin{array}{c}\text { Probability of } \\
\text { Occurrence } \\
\text { P(O) }\end{array}$} \\
\hline & S2 & S3 & \\
\hline Short & Discard & Discard & High \\
\hline Medium & Discard & Discard & Low \\
\hline Long & Discard & Discard & Very Low \\
\hline
\end{tabular}

Table 6. Fuzzy rules in case of one sensor failure

\begin{tabular}{|c|c|c|c|}
\hline \multicolumn{2}{|c|}{$\begin{array}{c}\text { Prediction } \\
\text { Success }\end{array}$} & \multirow{2}{*}{$\begin{array}{c}\text { Prediction_ } \\
\text { Failure } \\
\text { S3 }\end{array}$} & \multirow{2}{*}{$\begin{array}{c}\begin{array}{c}\text { Probability of } \\
\text { Occurrence }\end{array} \\
\mathbf{P}(\mathbf{O})\end{array}$} \\
\hline $\mathrm{S} 1$ & S2 & & \\
\hline Short & Short & Discard & High \\
\hline Medium & Short & Discard & High \\
\hline Long & Short & Discard & High \\
\hline
\end{tabular}

Table 7. Fuzzy rules in case of all sensors success

\begin{tabular}{|c|c|l|l|}
\hline \multicolumn{2}{|l|}{ Prediction_Success } & $\begin{array}{l}\text { Probability of } \\
\text { Occurrence }\end{array}$ \\
\hline S1 & S2 & S3 & P(O) \\
\hline Short & Short & Short & High \\
\hline Short & Medium & Medium & High \\
\hline Short & Long & Long & High \\
\hline
\end{tabular}

\subsection{Performance Evaluation by Fuzzy Inference Technique}

The ability to monitor the progress of unmanned boundary sensing is a critical issue. The present work aims to approach this problem by taking the benefit of fuzzy inference technique in order to classify elephant presence/detection according to the range $(\mathrm{S} / \mathrm{M} / \mathrm{L})$ of their location.

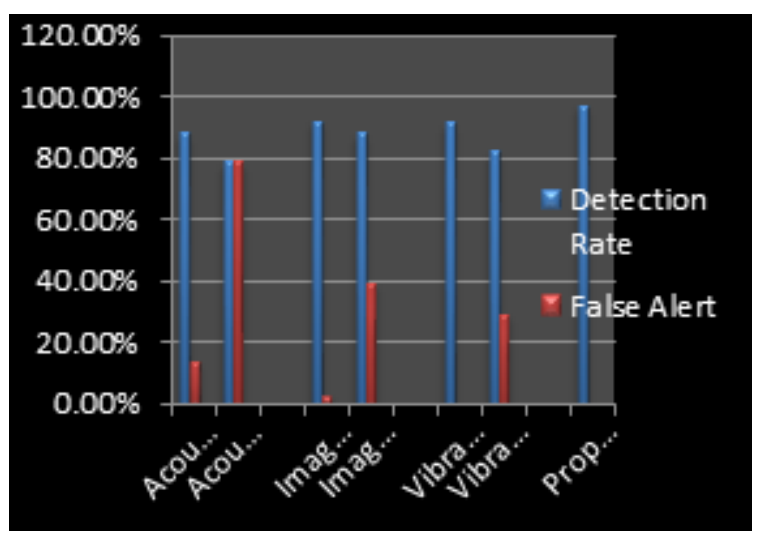

Figure 5. Performance Evaluation Graph

In this proposed approach we have performed fuzzification of the input data (distance metric of 
Table 8. Performance Evaluation

\begin{tabular}{|c|c|c|c|}
\hline \multicolumn{4}{|c|}{ Existing Methodologies } \\
\hline SI & Sensors & Experts & Performance \\
\hline \multirow{2}{*}{1} & \multirow{2}{*}{ Acoustic } & Zeppelzauer et al. (2015) & Detection Rate $88.2 \%$, False Positive rate $13.7 \%$ \\
\hline & & Hao et al. (2012) & Detection Rate $78.6 \%$, False Alert $78.6 \%$ \\
\hline \multirow[b]{2}{*}{2} & \multirow[b]{2}{*}{ Image } & Zeppelzauer et al. (2015)-Near & Detection Rate $91.7 \%$, False Positive Rate $2.5 \%$ \\
\hline & & Zeppelzauer et al. (2015)-Far & $\begin{array}{l}\text { Detection Rate } 88.0 \% \text {, False Positive Rate } \\
39.0 \%\end{array}$ \\
\hline \multirow{2}{*}{3} & \multirow{2}{*}{ Vibration } & S. J. Sugumar et al. (2013) & Accuracy $91.25 \%$, False Positive Rate $0 \%$ \\
\hline & & Jason et al. (2005) & Accuracy $82.0 \%$, False Positive Rate $29.0 \%$ \\
\hline \multicolumn{4}{|c|}{ Proposed Methodology } \\
\hline \multicolumn{3}{|c|}{$\begin{array}{l}\text { Integrated WSN based on Fuzzy Interference system } \\
\text { using Acoustic, Vibration \& Camera Sensors }\end{array}$} & $\begin{array}{l}\text { Detection Rate } 97.0 \% \\
\text { False Alert } 0 \%\end{array}$ \\
\hline
\end{tabular}

acoustic, vibration and image) by creating fuzzy inference system sensor-wise with/without failure.

For performance evaluation, we compare the proposed approach with two alternative existing approaches. The first approach is a method for acoustic detection introduced by Mattihas [7]. The second is a method for image detection introduced by Mattihas [7]. The novelty of the proposed integration process using fuzzy achieves a promising outcome. The results of all three evaluated methods along with the results of the proposed method achieves a 97.0\% detection rate with a $0 \%$ false alert as summarized in Table 8 and the graphical representation in Figure 5.

\section{Conclusion}

In conclusion, this paper provides a new intelligent architecture for elephant detection as a method and assists as a significant developing application for WSN and fuzzy system communal on the other. Three different framework developments where systematically integrated and the decision making is based on the most fundamental theory of fuzzy systems. The proposed system provides uses (forest authorities) with additional information to analyses distance metrics according to the location of elephants. Three different hardware prototypes have been used for this purpose, each of them with its own control. Further more systematic studies have been conducted in order to determine the accuracy of performance parameters (detection rate $\&$ false rate).

In future work the architecture would benefit from more advanced specifications in fuzzy systems. We believe this proposed work opens up superior chances for further theoretical and practical investigation using unmanned boundary sensing technology for different applications.

\section{REFERENCES}

1. Anni, J. S. \& Sangaiah,A. K. (2017). Wireless Integrated Sensor Network: Boundary Intellect system for elephant detection via cognitive theory and Fuzzy Cognitive Maps, Future Computer Generation Systems, doi. org/10.1016/j.future.2017.02.019.

2. Anni, D. J. S. \& Sangaiah, A. K. (2015). Elephant Tracking with Seismic Sensors: A Technical Perceptive Revie, Jurnal Teknologi, 74(1), 193-203.

3. Campana, B. J. L. \& Keogh, E. J. (2010). A compression-based distance measure for texture, Stat Anal Data Mining, 3(6), 381-398.

4. Cechak, J. (2010). Seismic Sensors Communication, AiMT Advances in Military Technology, 5(2), 1-17.

5. Gunther, R. H., O'Connell, R. \& Klempere, S. L. (2012). Seismic Waves from Elephant Vocalizations: A Possible Communication Mode, Geophys Res Lett, 31, L11602.

6. Hao, Y., Campana, B. \& Keogh, E. (2012). Monitoring and mining animal sounds in visual space, J Insect Behav, 26, 466-93.

7. Matthias, Z. (2013). Automated Detection of Elephants in Wildlife Video, EURASIP Journal on Image and Video Processing, 46(1), 1-23. 
8. Mayilvaganan, M. \& Devaki, M. (2014). Elephant Localization and Analysis of Signal Receiving in Base Station Using Acoustic Sensor Network, International Journal of Innovative Research in Computer and Communication Engineering, 2(2).

9. O'Connel-Rodwell, C. E., Wyman, M. T., Hart, B. T. \& Redfield, S. (2004). Interactive Patterns of Vocal Communication in African Elephant Herds (Loxodontaafricana), Journal of Acoustic Society of America, 115, 2555-65.

10. O'Connel-Rodwell, C. E., Arnason, E. \& Hart, B. T. (2001). Exploring the Potential Use of Seismic Waves as a Communication Channel by Elephants and Other Large Mammals, American Zoology, 41, 57-70.

11. Stoeger, A. S., Heimann, G., Zeppelzauer, M., Ganswindt, A., Hensman, S. \& Charlton, B. (2012). Visualizing sound emission of elephant vocalizations: evidence for two rumble production types, PLoS ONE, 7(11), e48907.
12. Sugumar, S. J \& Jayaparvathy, R. (2014). An improved real time image detection system for elephant intrusion along the forest border areas, The Scientific World Journal, 1-10. Article ID 393958.

13. Sugumar, S. J. \& Jayaparvathy, R. (2013). An early warning system for elephant intrusion along the forest border areas, Current Science, 104(11), 1515-1526.

14. Venter, P. J. \& Hanekom, J. J. (2010). Automatic detection of African elephant (Loxodonta Africana) infrasonic vocalizations from recordings, Biosyst Eng, 106(3), 286-294.

15. Zeppelzauer, M. \& Stoeger, A. (2015). Establishing the fundamentals for an elephant early warning and monitoring system, $B M C$ Res Notes, 8(409). DOI: 10.1186/s13104015-1370. 